\title{
COMPARAÇÃO ENTRE AS TÉCNICAS DE SHOULDICE E FALCI- LICHTENSTEIN, NO TRATAMENTO DAS HÉRNIAS INGUINAIS EM HOMENS
}

\author{
COMPARISON BETWEEN THE SHOULDICE AND FALCI-LICHTENSTEIN \\ TECHNIQUES ON THE TREATMENT OF INGUINAL HERNIAS IN MEN
}

\author{
José Luiz Campello de Mello Vianna, ACBC-MG ${ }^{1}$ \\ Alcino Lázaro da Silva, TCBC-MG ${ }^{2}$ \\ Antônio Sérgio Alves, TCBC-MG ${ }^{3}$ \\ Claudio Almeida de Oliveira ${ }^{4}$ \\ Álvaro Vieira Júnior ${ }^{5}$
}

\begin{abstract}
RESUMO: Objetivo: Trata-se de uma comparação, entre as técnicas Shouldice (S), um reparo em quatro camadas de sutura contínua e a de Falci-Lichtenstein (FL) que usa prótese de Polipropileno. Método: Foram operados 118 pacientes do sexo masculino, com um total de 124 hérnias, sendo que 58 pacientes foram submetidos a hernioplastia inguinal pela técnica de FL e 60 pacientes pela técnica de S. Em cada grupo foram reparadas 62 hérnias inguinais, com $85 \%$ dos pacientes acompanhados em quatro anos. A média de idade foi de 52 anos. Foram operadas 57 hérnias do tipo 3 A, 57 do tipo 3 B e 10 do tipo 4, segundo a classificação de Nyhus. Quanto ao lado houve uma predominância à direita com 65 hérnias $(52,4 \%)$. A anestesia foi epidural em $(89,8 \%)$ dos pacientes. Resultados: O hematoma e o seroma foram as complicações mais comuns no pósoperatório, seguidos pela retenção urinária e estas complicações ocorreram mais freqüentemente na técnica de FL. Houve uma recidiva $(0,8 \%)$ com a técnica de FL, em um paciente em que a tela soltou-se do ligamento inguinal. Em outro paciente apareceu uma hérnia femoral. Os pacientes operados pela técnica de S queixaramse mais de dor no pós-operatório imediato e retornaram mais tarde ao trabalho. O tempo de acompanhamento não foi o ideal, pois nos pacientes não controlados pode estar o maior índice de recidiva, quando se sabe que $40 \%$ das recidivas aparecem após cinco anos e $20 \%$ após 25 anos da operação primária. Conclusões: Os dois reparos apresentam taxas baixas de recidivas. A técnica de Shouldice é mais complexa, mas permite uma exploração segura do canal femoral, requer um conhecimento anatômico mais sólido da região e uma maior experiência com a cirurgia da hérnia inguinal. Seu custo é menor. O reparo Falci-Lichtenstein é eficaz, rápido, causa pouca dor, recuperação rápida e permite que os cirurgiões com menos experiência em cirurgia de hérnia inguinal possam realizá-la com sucesso.
\end{abstract}

Descritores: Hérnia inguinal; Canal Inguinal; Procedimentos cirúrgicos operatórios.

1. Professor Assistente de Técnica Operatória da Faculdade de Ciências Médicas de Minas Gerais; Mestrando em Cirurgia Geral pela Universidade Federal de Minas Gerais - UFMG; Cirurgião da Equipe de Cirurgia Geral II do Hospital Felício Rocho de Belo Horizonte - MG.

2. Professor Titular de Cirurgia do Aparelho Digestivo, Departamento de Cirurgia da Faculdade de Medicina da Universidade Federal de Minas Gerais.

3. Doutor em Cirurgia pela UFMG; Professor Adjunto de Técnica Operatória da Faculdade de Ciências Médicas de Minas Gerais; Cirurgião da Equipe de Cirurgia Geral II do Hospital Felício Rocho de Belo Horizonte - MG.

4. Professor de Técnica Operatória da Faculdade de Ciências Médicas de Minas Gerais; Chefe da Equipe de Cirurgia Geral II do Hospital Felício Rocho de Belo Horizonte - MG.

5. Professor Assistente de Técnica Operatória da Faculdade de Ciências Médicas Minas Gerais; Cirurgião da Equipe de Cirurgia Geral II Geral do Hospital Felício Rocho de Belo Horizonte; Clínica Cirúrgica II Belo Horizonte - MG.

Recebido em 17/09/2003

Aceito para publicação em 16/02/2003

Trabalho realizado no Hospital Felício Rocho e no Hospital Universitário São José da Faculdade de Ciências Médicas de MinasGerais. 


\section{INTRODUÇÃO}

A cirurgia da hérnia inguinal tem como finalidade abolir os defeitos congênitos ou adquiridos, da região inguinal, incluindo o anel inguinal profundo e a parede posterior do canal inguinal, preservando a anatomia, a função e a estética da região inguinal.

$\mathrm{O}$ reparo da hérnia inguinal, baseia-se no tratamento do saco herniário, do anel inguinal profundo (Marcy, 1871) $)^{1}$ e o reforço da parede posterior do canal inguinal (Bassini,1884) ${ }^{2-4}$. Com o emprego de próteses iniciou-se uma nova era da hernioplastia inguinal ${ }^{5}$.

A partir de 1945, em Toronto, Canadá, Dr. E. Earle Shouldice desenvolveu uma técnica para correção da hérnia inguinal. Esta técnica tem excelentes resultados, com uma taxa de recidiva de $1 \%{ }^{6,7}$.

A técnica consiste no reforço da parede posterior do canal inguinal, usando quatro camadas de sutura contínua com fio monofilamentar inabsorvível após dissecção adequada do anel inguinal interno. Pode ser aplicada para corrigir hérnias inguinais primárias, recidivadas, diretas, indiretas, combinadas.

Entre nós, Falci (1969) foi o primeiro a usar a tela de Márlex e publicou um trabalho com 100 pacientes, sendo um paciente com hérnia recidivada. A taxa de recidiva foi de $2 \%{ }^{8}$.

Em fevereiro de 1989, Irving I. Lichtenstein, publica sua técnica usando prótese de Polipropileno em 1.000 pacientes com hérnia primária com zero de recidiva, com cinco anos de acompanhamento'.

O objetivo deste estudo é comparar a técnica de Shouldice (S) com a técnica de Falci-Lichtenstein (FL).

\section{MÉTODO}

Neste estudo operamos 124 hérnias inguinais, em 118 pacientes, do sexo masculino, com idade entre 14 e 90 anos, no período de janeiro de 1997 a maio de 1999, no Hospital Felício Rocho e no Hospital Universitário São José da Faculdade de Ciências Médicas de Minas Gerais.

Dividimos os pacientes em dois grupos: um foi operado pela técnica de Shouldice (60 pacientes e 62 hérnias) e o outro grupo foi operado pela técnica de Falci-Lichtenstein (58 pacientes e 62 hérnias). A escolha do reparo a ser empregado foi por alternância. A média de idade dos pacientes operados pela técnica $\mathrm{S}$ foi de 52 anos e pela técnica FL foi de 57 anos.
O diagnóstico de hérnia indireta e direta foi igual $(45,9 \%)$ e das mistas foi de $(8,2 \%)$. No grupo operado pela técnica de FL, 32 hérnias eram do tipo direta $(51,6 \%)$ e 30 indiretas $(45,4 \%)$. Trinta e cinco pacientes tinham hérnia à direita $(60,3 \%)$ Nos pacientes que foram operados pela técnica de $\mathrm{S}$, a metade foi direta e a outra metade indireta. Quanto ao lado 32 hérnias $(51,6 \%)$ eram à esquerda. Os pacientes ASA $2(60,3 \%)$ predominaram no grupo FL e no grupo $S$ predominou ASA $1(56,6 \%)$.

$\mathrm{O}$ risco cirúrgico obedeceu à escala de estado físico da American Society Anesthesiologists (Tabela 1)

Tabela 1 - Risco Cirúrgico.

\begin{tabular}{ccc}
\hline CLASSE & $\mathbf{N}^{\mathbf{0}}$ DE PACIENTES & \% \\
\hline 1 & 51 & 43,2 \\
2 & 57 & 48,3 \\
3 & 10 & 8,5 \\
\hline
\end{tabular}

De acordo com a Escala de Estado Físico da "American Society Anesthesiologists" (ASA) dos 118 pacientes.

Como causas de hérnia inguinal o esforço físico estava presente em $16,1 \%$ e a história familiar em $24,6 \%$ dos pacientes. A hérnia redutível correspondeu a $93,5 \%$ dos pacientes e nestes foram operadas simultâneamente seis hérnias umbilicais e três epigástricas.

Quanto ao tipo e o lado, predominaram as do tipo direta $(50,8 \%)$ e o lado direito $(52,4 \%)$ respectivamente. A classificação de Nyhus foi adotada.

A anestesia foi a epidural em 106 pacientes $(89,8 \%)$.

A inguinotomia foi arqueada, com $10 \mathrm{~cm}$ aproximadamente de comprimento e acompanhando as linhas de força da região, a mais ou menos $2 \mathrm{~cm}$ do ligamento inguinal; o isolamento do cordão espermático com Penrose número 2 e abertura das túnicas do funículo, do cremaster e a ressecção dos lipomas foram semelhantes nas duas técnicas.

O saco herniário das hérnias indiretas foram tratados com ligadura acima do anel inguinal profundo nos dois reparos. Nas hérnias diretas o tratamento do saco, na técnica $\mathrm{S}$, foi redução da gordura préperitoneal e ressecção da parte enfraquecida da fáscia transversal. Na FL o saco herniário foi reduzido com uma sutura em bolsa. 


\section{Reparo à Shouldice (S)}

A fáscia transversal foi aberta do anel inguinal profundo até o púbis a mais ou menos $2 \mathrm{~cm}$ do ligamento inguinal. Tanto o retalho ínfero-lateral quanto o retalho súpero-medial, foram reparados e liberados da gordura pré-peritoneal de modo a expor, superiormente, a borda lateral da bainha do músculo reto do abdome e, inferiormente, até a região femoral que foi explorada digitalmente.

\section{Primeiro plano do reparo}

$\mathrm{O}$ reforço foi feito com fio prolene 2-0 e começou junto ao tubérculo púbico e o ponto foi dado na borda livre do retalho ínfero-lateral da fáscia transversal, sem atingir o periósteo, que foi suturada debaixo do retalho súpero-medial da fáscia transversal e amarrada sem tensão e uma extremidade curta do fio foi deixado para posterior utilização. A sutura inclui a borda lateral da bainha do músculo reto do abdome, seguindo em direção ao anel inguinal profundo. Lateralmente a sutura inclui uma superfície profunda da camada formada pelos músculos oblíquo interno, transverso e pela fáscia transversal até criar um novo anel inguinal profundo. Esta camada tem pouca resistência, mas permite que a fáscia transversal funda-se à bainha femoral e contém o peritônio e a gordura préperitoneal (Figura1).

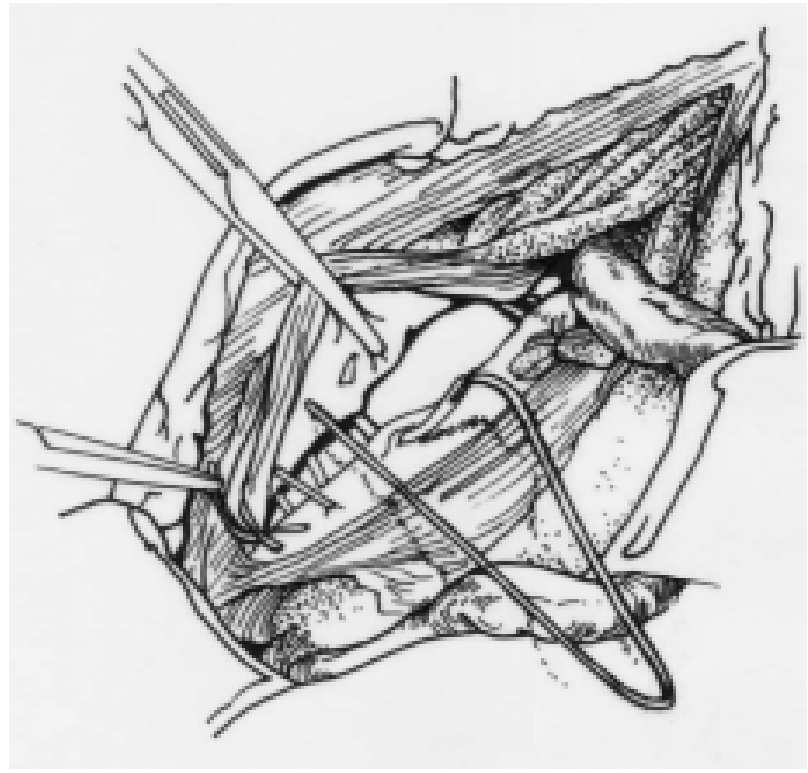

Figura 1 - Sutura borda lateral da bainha do MRA e primeiro plano de sutura.

\section{Segundo plano do reparo}

Utilizou-se a mesma sutura contínua, no sentido do anel inguinal profundo até o púbis. O retalho súperomedial da fáscia transversal é suturado ao ligmento inguinal e a sutura é amarrada na extremidade do fio que foi preservado na primeira linha de sutura além do nó inicial, e neste ponto o fio é cortado (Figura 2).

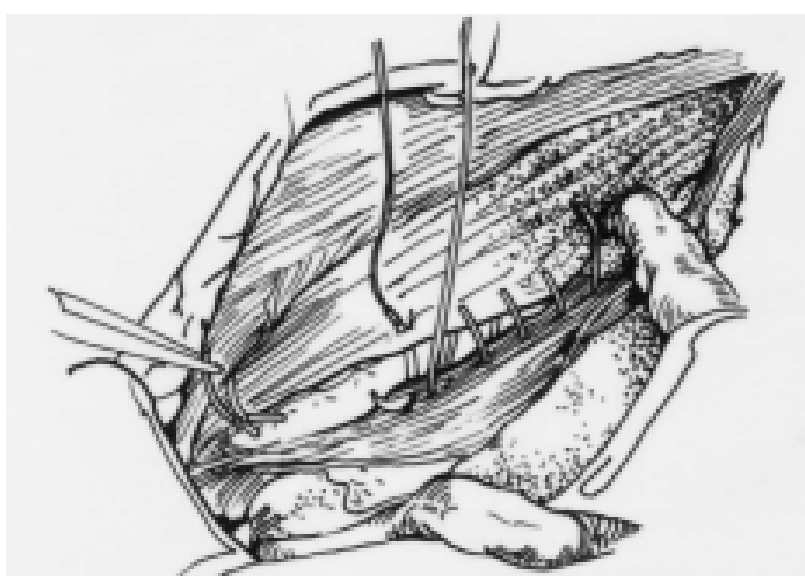

Figura 2 - Segundo plano de sutura.

\section{Terceiro plano do reparo}

Usou-se o mesmo fio, começando bem junto ao anel inguinal profundo, suturando-se a superfície do músculo oblíquo interno à superficie inferior da aponeurose ínfero-lateral do músculo oblíquo externo próximo ao ligamento inguinal até o púbis.

\section{Quarto plano do reparo}

Com o mesmo fio, a sutura vai do púbis até o anel inguinal profundo, reforçando o plano anterior e terminando junto ao anel inguinal profundo, onde o fio foi anodado (Figura 3)

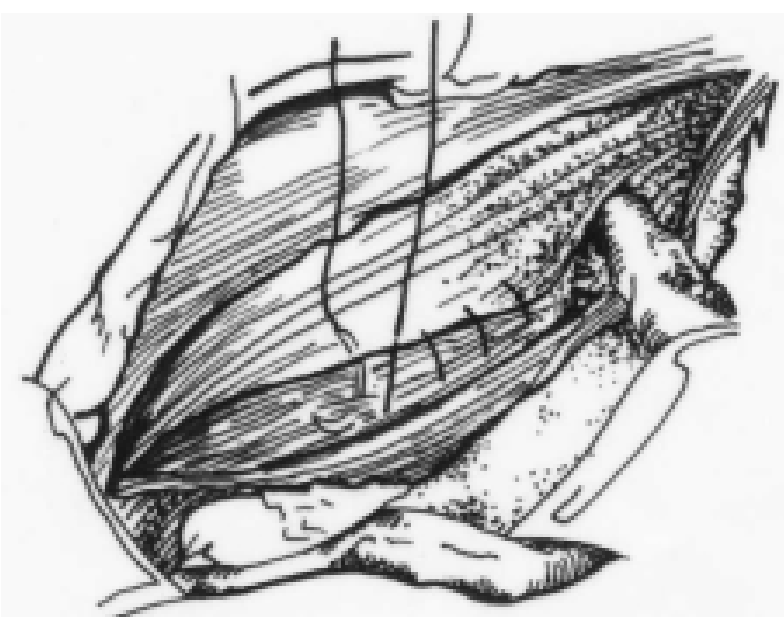

Figura 3 - Quarto plano de sutura. 


\section{Reparo Falci-Lichtenstein (FL)}

Uma tela de Polipropileno (Márlex), previamente preparada, foi modelada de acordo com a região inguinal a ser operada. A tela foi fixada a $2 \mathrm{~cm}$ além do tubérculo púbico com três pontos separados de prolene $2-0$, porque a tela sofre retração e não deixa a região desprotegida, pois, a recidiva é mais comum neste local.

A seguir a tela é suturada inferiormente ao ligamento inguinal com sutura contínua de Prolene 20 até $\mathrm{o}$ anel inguinal profundo; medialmente à bainha do músculo reto do abdome; superiormente na aponeurose do músculo oblíquo interno ou sob o folheto superior da aponeurose do músculo oblíquo externo, com sutura de Poliglactina 910, 2-0 com pontos separados (Figuras 4 e 5).

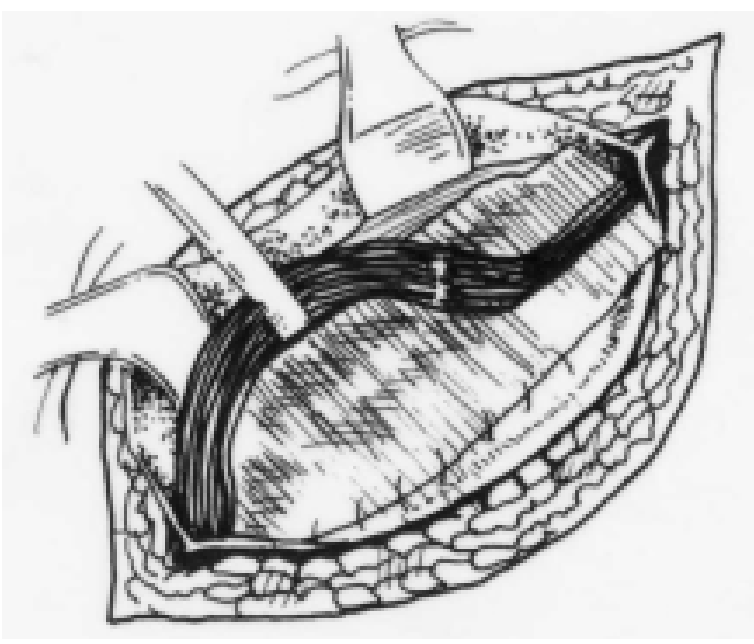

Figura 4 - Sutura da tela ao ligamento inguinal.

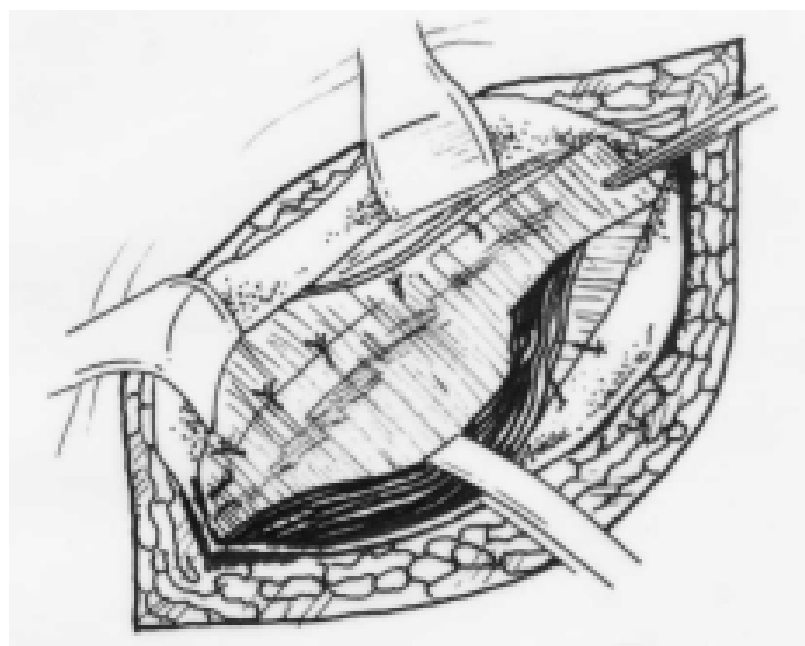

Figura 5 - Sutura da tela medialmente, superiomente e o novo anel interno.
Foi feito, uma fenda na tela, próximo ao anel inguinal profundo, para permitir a saída do cordão espermático, criando dois segmentos, sendo que a parte lateral representa $1 / 3$ da tela e a medial 2/3. Estes segmentos cruzam ao redor do cordão, de maneira que o segmento medial, que é mais largo, passa por cima do cordão e é fixado ao segmento mais estreito e os dois são suturados ao ligamento inguinal com um ponto simples de Prolene 2-0. Este procedimento, cria um novo anel interno e um mecanismo obturador (Figura 5).

A tela é colocada frouxa, pois $20 \%$ a $30 \%$ sofre retração e pode soltar-se da região inguinal, propiciando uma recidiva da hérnia.

No fechamento da aponeurose de músculo oblíquo externo foi empregado a Poliglactina 910, 3-0, nas fáscias de Camper e Scarpa e na tela subcutânea usamos o Categute, 3-0 e na pele foi usado mononáilon 4-0. Este procedimento foi igual nos dois grupos.

A movimentação ativa e deambulação precoce foram estimuladas no pós-operatório imediato e o curativo oclusivo ficou por 24 horas.

\section{RESULTADOS}

As complicações pós-operatórias imediatas ocorreram em 22 pacientes (37,9\%) do grupo FL e em 18 pacientes (30\%) do grupo S sem necessidade de tratamento cirúrgico, sendo o hematoma e o seroma os mais comuns. A retenção urinária, a orquite, dor por mais de três meses $(8,6 \%)$, equimose, edema de escroto foram mais freqüentes nos pacientes do grupo FL (Tabela 2)

O tempo médio de internação foi de 1,75 dias e após a alta hospitalar, $85 \%$ destes pacientes foram controlados com um mês, três meses, e depois de três em três meses até 48 meses. Houve no pós-operatório, uma recidiva, pela técnica de FL (a tela soltou-se do ligamento inguinal) e em outro paciente apareceu uma hérnia femoral. Óbitos ocorreram por outras causas, durante o acompanhamento tardio.

\section{DISCUSSÃO}

O reforço da parede posterior do canal inguinal sempre foi uma preocupação para os cirurgiões e vários tipos de enxertos como: pele, aponeurose, aço inoxidável, próteses metálicas, nailon, órlon, polietileno entre outros foram introduzidos e testados para reforçar a parede posterior do canal inguinal ${ }^{5,8}$. 
Tabela 2 - Complicações Pós-Operatórias Imediatas.

\begin{tabular}{lcc}
\hline COMPLICAÇÕES & SHOULDICE & FALCI-LICHTENSTEIN \\
\hline Hematoma e Seroma & 4 & 10 \\
Retenção Urinária & 4 & 3 \\
Orquite & 0 & 1 \\
Complicações Respiratórias & 2 & 0 \\
Outras & 8 & 8 \\
Sangramento & 2 & 2 \\
Edema & 4 & 1 \\
Equimose & 2 & 5 \\
\hline
\end{tabular}

A correção cirúrgica das hérnias inguinais tanto pela técnica de Shouldice quanto pela técnica de Falci-Lichtenstein tem mostrado baixos índices de recidivas e de complicações. Estes reparos permitem a deambulação precoce, podem ser usadas em hérnias primárias e recidivadas. Quanto a classificação de Nyhus são empregadas nas hérnias tipo 3A, 3B e $4^{10}$.

$\mathrm{O}$ reparo à Shouldice permite que toda região inguino-femoral seja explorada e apesar disso, é uma técnica pouca difundida em nosso meio.

Em um estudo prospectivo e randomizado, Hay et al. compararam as técnicas de Shouldice, Bassine e McVay em 1.578 pacientes com hérnias de diferentes tipos e de acordo com os resultados, consideraram o reparo à Shouldice como "padrão-ouro" para estudos comparativos entre outras técnicas para hernioplastia inguinal ${ }^{11}$.

Glassow et $a l^{3}$. compararam a técnica de Shouldice com o reparo da parede inguinal posterior usando-se o ligamento de Cooper. Conseguiram taxas de recidivas de $0,7 \%$ para reparo à Shouldice contra $4,9 \%$ para reparo usando o ligamento de Cooper.

Wantz ${ }^{6}$, empregando o reparo de Shouldice operou de 1970 a 1987, 4.366 hérnias primárias com $1,3 \%$ de recidiva.

Devlin et $a l^{4}$. repararam 718 hérnias com a técnica de Shouldice em 696 pacientes e relataram a probabilidade de recorrência da hérnia inguinal em aproximadamente $1 \%$ em 10 anos.

Entre nós, podemos citar Costa e Silva et al ${ }^{12}$. que operaram 1.034 hérnias inguinais pela técnica de Shouldice, sendo 102 hérnias recidivadas. Relatam um índice de recorrência de $0,8 \%$ para as hérnias primárias e 4,9\% para as recidivadas ${ }^{9,12}$. Melo et al ${ }^{13}$. em 115 hérnias primárias, tiveram uma taxa de recidiva de $1,7 \%$, em oito anos de acompanhamento.
A prótese de Polipropileno (Márlex), foi usada pela primeira vez em 1958 por Usher ${ }^{8}$.

No nosso meio Falci (1969) foi o primeiro a empregar a tela de Márlex, em 100 pacientes, com 113 reparos e $2 \%$ de recidiva ${ }^{8}$.

O método foi publicado por Lichtenstein em 1989 e amplamente divulgado. Historicamente, pois, é justo que o epônimo seja FL.

Com o uso das próteses modernas, é possível reparar todas as hérnias inguinais sem distorção da anatomia e sem tensão na linha de sutura.

A prótese ideal seria aquela que não se modificasse com os fluídos dos tecidos, fosse quimicamente inerte, não estimulasse a inflamação, não criasse reação de corpo estranho e não fosse carcinogênica ${ }^{5}$.

Considerando a fáscia transversal um envoltório fino, de pouca resistência na sua maior extensão, com poucas fibras aponeuróticas, Lichtenstein, em 1989, publica sua técnica (livre de tensão) em 1.000 pacientes com hérnia inguinal primária, nos quais usou a prótese de Polipropileno, com acompanhamento de cinco anos e zero de recidiva ${ }^{9}$.

Ele fez várias considerações sobre o emprego da tela no reforço da parede posterior do canal inguinal: não confiava na fáscia transversal, o tendão conjunto era um mito, o reparo da região com tecido enfraquecido poderá levar ao insucesso, sutura sob tensão seria a principal causa de recidiva precoce e distúrbios metabólicos do colágeno na fáscia transversal seria causa de recidiva tardia ${ }^{1}$.

O saco herniário indireto, nesse reparo, é dissecado e reduzido sem ser ligado. $\mathrm{O}$ saco da hérnia direta é reduzido com uma sutura em bolsa. A tela é fixada livre de tensão sobre a fáscia transversal, evitando que ela se solte dos pontos de fixação e consequentemente propiciando a recorrência da hérnia ${ }^{1}$. 
Amid et $a l^{14}$. relatam vantagens da anestesia local no tratamento de 12.000 hernioplastias inguinais e atribuem a erros técnicos as recorrências com esta técnica. Operaram, entre junho de 1984 a junho de 1995, 4.000 hérnias inguinais primárias, sendo 1.000 bilaterias com uma taxa de recidiva de $0,1 \%{ }^{14,15}$.

Lichtenstein em vários artigos confirma a baixa taxa de recorrência com esta técnica ${ }^{9,14-16}$.

Shulman et $\mathrm{l}^{16}$. em 6.321 pacientes adultos com hérnias inguinais, demonstraram diminuição da dor no pós-operatório, quando não ligaram e nem ressecaram o saco herniário.

Wantz $^{17}$ em seu trabalho com 1.252 hernioplastias, relata seis recidivas $(0,5 \%)$ com seis anos de acompanhamento.

No reparo FL, os pacientes queixaram menos no pós-operatório imediato e retornaram mais pre- cocemente ao trabalho. O hematoma e o seroma foram mais comuns neste tipo de reparo.

Na técnica de Shouldice os pacientes não tiveram complicações como orquite e edema de escroto. A retenção urinária esteve presente nos pacientes operados indiferentemente da técnica e estava relacionada ao tipo de anestesia.

O tempo cirúrgico e a permanência hospitalar foram maiores no reparo à Shouldice, respectivamente, 20 minutos e 12 horas.

O custo é menor com a técnica de Shouldice, levando em conta o uso de menos fios e que não emprega prótese.

Com base na experiência, no baixo índice de complicações e de recidivas, pode-se recomendar estas duas técnicas para a correção cirúrgica das hérnias inguinais do tipos $3 \mathrm{~A}, 3 \mathrm{~B}$ e 4 segundo a classificação de Nyhus.

\begin{abstract}
Objective: We compared the Shouldice (S) technique, a repair in four layers of continuous suture and the Falci-Lichtenstein (FL) technique that uses polypropylene prothesis. Method: One hundred and eighteen male patients underwent surgery, on a total of 124 hernias. Fifty-eight of them underwent inguinal hernia surgery by the FL technique, and the other 60 patients by the $S$ technique. In each group 62 inguinais hernias with $85 \%$ of the patients were followed for four years. The average age was 52 years old. Fifty-three type 3 A hernias, 57 type $3 B$ and 10 type 4 hernias were operated, according to the Nyhus classification. Concerning the side, there was a predominance on the right with 65 ruptures $(52.4 \%)$. The anesthesia was spinal in (89.8\%) of the patients. Results: The bruise and the seroma were the most common postoperative complications, occuring more frequently with the FL technique. In this one there was a recurrence $(0,8 \%)$, because the bandage and the inguinal ligament broke up. In another patient, a femoral rupture turned up. The patients operated through the S technique complained more about pain on the immediate postoperative period and returned to work later. The observation time wasn't the ideal, because the largest index of recurrence can be in the non controlled patients, as it's known that $40 \%$ of the recurrence occur 5 years after the primary operation and 20\% 25 years after. Conclusions: Both repairs presented low rates of recurrence. The Shouldice technique is more complex, as it allows a safe exploration of the femoral channel, requires a more solid anatomical knowledge of the region and a better experience with the surgery of the inguinal hernia and it's a low cost procedure. The Falci-Lichtenstein repair is effective,rapid, causes fewer pain, fast recovery and allows less experienced inguinal hernia surgeons to accomplish it successfully.
\end{abstract}

Key Words: Hernia, inguinal; Inguinal canal; Surgical procedures, operative.

\section{REFERÊNCIAS}

1. Griffith CA. - A correção de Marcy revista. Clin Cir Am Norte, 1984, 2: 229-242.

2. Lichtenstein IL, Shore JM - Exploding the myths of hernia repair. Am J Surg, 1976, 132(3):307-315.

3. Glassow F - Inguinal hernia repair. A comparison of the Shouldice and Cooper ligament repair of the posterior inguinal wall. Am J Surg, 1976, 131(3):306-311.
4. Devlin HB, Gillen PH, Waxman BP, et al. - Short stay surgery for inguinal hernia: experience of the Shouldice operation, 1970-1982. Br J Surg, 1986, 73(2):123-124.

5. DeBord JR. - O desenvolvimento histórico das próteses na cirurgia de hérnia. Clin Cir Am Norte, 1998, 6: 57-93.

6. Wantz GE - The Canadian repair: personal observations. World J Surg, 1989, 13(5):516-521.

7. Glassow F - The Shouldice Hospital technique. Int Surg, 1986, 71(3):148-153. 
8. Falci F - Marlex-mesh no tratamento da hérnia inguinal do adulto (análise de 100 casos operados). Hospital, 1969, 75(1):147-159.

9. Lichtenstein IL, Shulman AG, Amid PK, et al. - The tension-free hernioplasty. Am J Surg, 1989, 157(2):188193.

10. Nyhus LM - Individualization of hernia repair: a new era. Surgery, 1993, 114(1):1-2.

11. Hay JM, Boudet MJ, Fingerhut A, et al. - Shouldice inguinal hernia repair in the male adult: the gold standard? A multicenter controlled trial in 1578 patients. Ann Surg, 1995, 222(6):719-727.

12. Silva NC, Reis MC, Candido-Lima AP, et al. - Reparo da hérnia inguinal pela técnica de Shouldice. Rev Hosp Clin Fac Med São Paulo, 1995, 50(6):314-316.

13. Melo RM, Cozadi AO, Matias IS, et al. - Reparo pela técnica de Shouldice modificada nas hérnias inguinais primárias. Rev Col Bras Cir, 1998, 25(3): 167-171.
14. Shulman AG, Amid PK, Lichtenstein IL - The safety of mesh repair for primary inguinal hernias: results of 3,019 operations from five diverse surgical sources. Am Surg, 1992, 58(4):255-257.

15. Amid PK, Shulman AG, Lichtenstein IL - Local anesthesia for inguinal hernia repair step-by-step procedure. Ann Surg, 1994, 220(6):735-737.

16. Shulman AG, Amid PK, Lichtenstein IL - Ligation of hernial sac. A needless step in adult hernioplasty. Int Surg, 1993, 78(2):152-153.

17. Wantz GE - Experience with the tension-free hernioplasty for primary inguinal hernias in men. J Am Coll Surg, 1996, 183(4):351-356.

Endereço para correspondência:

Alcino Lázaro da Silva

Rua Guaratinga, 151- Sion

30315-430- Belo Horizonte - MG 